\title{
Re-examination of the thalamostriatal projections in the rat with retrograde tracers
}

\author{
M. Elena Erro, José L. Lanciego, José M. Giménez-Amaya* \\ Departamento de Anatomía, Facultad de Medicina, Universidad de Navarra, C/Irunlarrea, 31008 Pamplona, Navarra, Spain
}

Received 11 May 2001; accepted 11 October 2001

\begin{abstract}
Topographical arrangements of thalamostriatal projection neurons was examined in the rat by the retrograde tract-tracing method. After injecting Fluoro-Gold (FG) and/or cholera toxin $\beta$-subunit (CTB) in different regions of the caudate-putamen $(\mathrm{CPu})$, distribution of retrogradely labeled neurons was observed in the thalamus. The main findings were as follows: (1) Retrogradely labeled neurons were seen in the midline-intralaminar thalamic nuclei in all rats examined in the present study. Neurons in the ventral, lateral and posterior thalamic nuclear groups were also labeled in the rats which were injected with the tracer into the dorsal part of the $\mathrm{CPu}$, but not in the rats which were injected with the tracer into the nucleus accumbens (Acb) and its adjacent regions in the ventromedial part of the $\mathrm{CPu}$. (2) Topographical organization was observed in the projections from the midline-intralaminar thalamic nuclei to the $\mathrm{CPu}$. After the tracer injection into the dorsal part of the $\mathrm{CPu}$ or the ventral part of the $\mathrm{CPu}$ (including the Acb), labeled neurons in the midline-intralaminar thalamic nuclei were distributed predominantly in the lateral part of the intralaminar nuclei or the midline nuclei, respectively. On the other hand, after the tracer injection into the medial or the lateral part of the $\mathrm{CPu}$, labeled neurons in the midline-intralaminar nuclei were distributed mainly in the dorsal or the ventral part of these nuclei, respectively. (3) Topographical organization was also observed in the thalamostriatal projections from the ventral and Pos. After the tracer injection into the rostral part of the $\mathrm{CPu}$, labeled neurons were distributed mainly in the rostral part of the ventral nuclear group. On the other hand, after the tracer injection into the caudal part of the $\mathrm{CPu}$, labeled neurons were distributed mainly in the caudal part of the ventral nuclear group, as well as in the posterior nuclear group. (C) 2002 Published by Elsevier Science Ireland Ltd. and the Japan Neuroscience Society.
\end{abstract}

Keywords: Basal ganglia; Striatum; Thalamus; Fluoro-Gold; Cholera toxin $\beta$-subunit

\section{Introduction}

Recently, particular attention has been paid to the thalamus as an important area for interaction between the input and the output systems of the basal ganglia through the thalamostriatal projections (Heras et al., 1997, 1998b; Erro et al., 1999; Giménez-Amaya and Scarnati, 1999; Lanciego et al., 1999; Mengual et al., 1999; Giménez-Amaya et al., 2000). Although this pathway has been studied in several animal species,

Abbreviations: Acb, accumbens nucleus, core; AD, anterodorsal thalamic nucleus; APTD, anterior pretectal nucleus, dorsal part; APTV, anterior pretectal nucleus, ventral part; CL, centrolateral thalamic nucleus; CM, central medial thalamic nucleus; Cpu, caudate putamen; Eth, ethmoid thalamic nucleus; F, nucleus of the fields of Forel; fr, fasciculus retroflexus; IAM, interanteromedial thalamic nucleus; IMD, intermediodorsal thalamic nucleus; LD, laterodorsal thalamic nucleus; LHb, lateral habenular nucleus; LP, lateral posterior thalamic nucleus; MD, mediodorsal thalamic nucleus; MHb, medial habenular nucleus; ml, medial lemniscus; mt, mammillothalamic tract; OPC, oval paracentral thalamic nucleus; PC, paracentral thalamic nucleus; PF, parafascicular thalamic nucleus; Po, posterior thalamic nuclear group; PoMn, posteromedian thalamic nucleus; PR, prerubral field; PV, paraventricular thalamic nucleus; Re, reuniens thalamic nucleus; Rh, rhomboid thalamic nucleus; RI, rostral interstitial nucleus of the medial longitudinal fasciculus; Rt, reticular thalamic nucleus; sm, stria medullaris of the thalamus; spc, superior cerebellar peduncle; SPF, subparafascicular thalamic nucleus; SPFPC, subparafascicular thalamic nucleus, parvicellular part; Sub, submedius thalamic nucleus; VL, ventrolateral thalamic nucleus; VM, ventromedial thalamic nucleus; VPM, ventral posteromedial thalamic nucleus; VPL, ventral posterolateral thalamic nucleus; VPPC, ventral posterior thalamic nucleus, parvicellular part; ZI, zona incerta.

* Corresponding author. Tel.: + 34-948-425-685; fax: + 34-948-425-649.

E-mail address: jmga@unav.es (J.M. Giménez-Amaya). 
retrograde tract-tracing studies in the rat using modern tracer techniques have not been carried out.

The thalamostriatal fibers of the rat originates predominantly from the intralaminar and midline thalamic nuclei (Nauta et al., 1974; van der Kooy, 1979; Newman and Winans, 1980; Veening et al., 1980; Hazlett and Bagley, 1983; Phillipson and Griffiths, 1985; Fuller et al., 1987; Christie et al., 1987; Berendse and Groenewegen, 1990; Erro et al., 2001). Thalamostriatal fibers from other thalamic nuclei has also been described in the rat, such as that from the mediodorsal thalamic nucleus (MD) (Veening et al., 1980; Christie et al., 1987; Fuller et al., 1987; Groenewegen, 1988), from a group of neurons placed lateral to the stria medullaris (Phillipson and Griffiths, 1985), from medial areas of the ventromedial thalamic nucleus (VM), from the posterior nuclear group (Berendse and Groenewegen, 1990) and from the medial subdivision of the geniculate thalamic nucleus (Veening et al., 1980; Lin et al., 1984).

Retrograde tract-tracing studies have defined a topographic organization for the thalamostriatal system of the rat. van der Kooy (1979) described that dorsomedial areas of the thalamus project to the medial region of the caudate-putamen $(\mathrm{CPu})$, while the lateral $\mathrm{CPu}$ is reached by neurons located in ventrolateral areas of the thalamus. Veening et al. (1980) demonstrated that the thalamostriatal system displayed a topographical organization that followed the rostrocaudal axis, in such a way that rostral thalamic nuclei project preferentially to the rostral $\mathrm{CPu}$ and vice versa. Recently Berendse and Groenewegen (1990) published a more detailed study of the organization of the thalamostriatal system of the rat by injecting the anterograde tracer Phaseolus vulgaris leucoagglutinin into individual midline and intralaminar nuclei. The terminal fields of these thalamic nuclei were described as obliquely oriented zones with a large rostrocaudal extent, with the midline nuclei projecting to the medial and ventral areas and the intralaminar nuclei shifting gradually to the lateral and dorsal areas of the $\mathrm{CPu}$.

The aim of the present study was to re-examine in detail the organization of the thalamostriatal projections by combining two different retrograde tracers: Fluro-Gold (FG) and cholera toxin $\beta$-subunit (CTB). For this purpose, small injections of FG and CTB were placed into different locations of the $\mathrm{CPu}$ in an attempt to fill the entire structure. The localization of the retrogradely labeled neurons within the thalamic nuclei was analyzed accurately using adjacent Nissl and acetylcholinesterase (AChE) stained sections.

\section{Material and methods}

Female Wistar rats $(n=30$; body weight ranging from 200 to $230 \mathrm{~g}$ ) were used in this study. Animals were deeply anesthetized $(0.1 \mathrm{ml} / 100 \mathrm{~g})$ with an intramuscular injection of Ketaset $^{\circledR}$ (1\% ketamine solution) and $\operatorname{Rompun}^{\circledR}(2 \%$ xylacine solution) at $4: 3$ ratio, and placed in a stereotaxic frame. A $2 \%$ solution of FG (Fluorochrome, Englewood, CO) in $0.1 \mathrm{M}$ cacodylate buffer ( $\mathrm{pH}$ 7.3) was iontophoretically injected into the $\mathrm{CPu}$ of 27 animals through a glass micropipette (inner tip diameter of $40-60 \mu \mathrm{m})$ using a $5 \mu \mathrm{A}$ positive-pulsed direct current ( $7 \mathrm{~s}$ on/off for $5 \mathrm{~min}$ ). In one surgical session, three animals were injected with $0.5 \mu \mathrm{l}$ of a solution of $2 \% \mathrm{CTB}$ in $0.125 \mathrm{M}$ phosphate buffer (PB) (List Biological Laboratories, Campbell, CA) in the ipsilateral $\mathrm{CPu}$ using a Hamilton syringe. Stereotaxic coordinates were taken from the atlas of Paxinos and Watson (1998). At all times, animals were handled according to the Society for Neuroscience Policy on the Use of Animals in Neuroscience Research as well as the European Communities Council Directive 86/609/EEC.

After a survival time of 7 days, the animals were anesthetized with an intraperitoneal overdose of $10 \%$ chloral hydrate in distilled water and perfused transcardially. Perfusion was carried out with a saline rinsing solution at body temperature, immediately followed by $500 \mathrm{ml}$ of cold fixative containing $4 \%$ paraformaldehyde, $0.1 \%$ glutaraldehyde and $0.2 \%$ saturated picric acid in $0.125 \mathrm{M} \mathrm{PB}(\mathrm{pH} 7.4)$. After the perfusion, the skull was opened and the brain removed and stored in a cryoprotective solution containing 2\% dimethylsulphoxide and $20 \%$ glycerin in $0.125 \mathrm{~PB}(\mathrm{pH} 7.4)$ (Rosene et al., 1986). Coronal frozen sections (40 $\mu \mathrm{m}$ thick) were obtained using a sliding microtome and collected in $0.125 \mathrm{M} \mathrm{PB}$ (pH 7.4). A total number of ten series of sections in each brain was obtained. One out of ten sections was processed for tracer visualization.

We will only describe the staining protocol conducted for the simultaneous detection of retrogradely transported CTB and FG. Only minor differences may be observed when trying to stain transported FG in singletracing experiments. Sections were incubated in a cocktail solution of primary antisera comprising 1:2000 goat anti-CTB (List Biological Laboratories, Campbell, CA) and 1:2000 rabbit anti-FG (Chemicon, Temecula, CA) antibodies for $60 \mathrm{~h}$ at $4{ }^{\circ} \mathrm{C}$ with gentle agitation. Incubation was continued in a second cocktail solution of bridge antisera containing 1:50 donkey anti-goat IgG (Nordic Immunological Laboratories, Tilburg, The Netherlands) and 1:50 swine anti-rabbit IgG (Dako, Copenhagen, Denmark) for $120 \mathrm{~min}$ at room temperature. Sections were then incubated in a peroxidase-antiperoxidase (PAP) complex raised in goat (1:600 goat-PAP, Nordic) for $90 \mathrm{~min}$ at room temperature. The CTB protocol was completed within 10-20 min using diaminobenzidine (DAB; Sigma Chemical CO., St. Louis, MO) as the chromogen for detecting the peroxidase reaction. The final step consisted of incuba- 
tion with a PAP complex raised in rabbit (1:600 rabbitPAP) (Dako, Copenhagen, Denmark) for $90 \mathrm{~min}$ followed by a reaction for 5-10 min using a Vector $^{\circledR}$ VIP peroxidase substrate (V-VIP, Vector, Burlingame, CA) resulting in the formation of a purple precipitate. Extensive washing with $0.05 \mathrm{M}$ Tris-buffered saline (TBS, $\mathrm{pH}$ 8.0 ) with $0.5 \%$ Triton $\mathrm{x}-100$ (TBS-Tx) was carried out throughout the procedure. Rinsing several times with $0.05 \mathrm{M}$ Tris $-\mathrm{HCl}$ (pH 7.6) were also conducted before and after the incubation in the chromogen solution. The primary antiserum was prepared in TBS-Tx ( $\mathrm{pH} 8.0$ ) with $0.2 \%$ bovine serum albumin (Merck, Darmstadt, Germany) to prevent non specific binding. After the staining was completed, the sections were mounted on glass slides using a solution of $2 \%$ gelatin (Merck, Darmstadt, Germany) in 0.05 M Tris$\mathrm{HCl}(\mathrm{pH}$ 7.6) dried, rapidly dehydrated in toluene, and coverslipped with Entellan ${ }^{\circledR}$. Adjacent sections were processed for AChE (Geneser-Jensen and Blackstad, 1971; Graybiel and Ragsdale, 1978) and Nissl-stained with thionin.

The exact locations of the injection sites and the retrogradely labeled neurons in the thalamus were directly drawn using an image taken with a camera lucida. Each drawing displaying labeled neurons was then scanned into a computer (Power Macintosh 9500, Apple, Spain) and both the thalamic contours and the structures displaying labeled neurons were redrawn using Freehand 7.0.1 (Macromedia, San Francisco, CA). In order to better delineate the different thalamic nuclei for determining the exact location of the retrogradely labeled neurons, the adjacent Nissl- and AChE-stained series were used as a reference.

\section{Results}

\subsection{Injection sites into the $\mathrm{CPu}$}

The retrograde tracers FG and CTB were injected into the $\mathrm{CPu}$ at different stereotaxic coordinates trying to cover as much striatal tissue as possible. A total of 30 FG injection sites were included in this study. Single-FG injections were carried out in 27 cases, and in three cases, FG was injected concomitantly with CTB (cases 98085 , 98050 and 98028 ) (Fig. 5). No leakage along the injection tract contaminating the cortex was observed in any of the cases. Twenty-one FG injection sites and the three CTB injection sites were located in the precommissural $\mathrm{CPu}$, rostrally to the decussation of the anterior commissure. In two rats, the nucleus accumbens (Acb) was involved in the FG injection sites in the ventrolateral part of the $\mathrm{CPu}$. Nine FG injection sites were located in the postcommissural $\mathrm{CPu}$. Fig. 1 summarizes the location of all of the tracer injection sites.

\subsection{Distribution of retrogradely labeled neurons in the thalamus}

After the striatal injections of FG, retrogradely labeled neurons were always found in the ipsilateral midline and intralaminar thalamic nuclei irrespective of the location of the FG injection site. Retrogradely labeled neurons were found in the ventral (VM, ventrolateral (VL), ventral posteromedial thalamic nuclei(VPM) and ventral posterolateral thalamic nuclei (VPL)), lateral (laterodorsal (LD) and lateral posterior (LP) thalamic nuclei) and posterior thalamic nuclear groups (Po) as a function of the rostrocaudal location of the injection sites in the $\mathrm{CPu}$. The neurons located in the midline and intralaminar thalamic nuclei displayed a cluster like distribution whereas the neurons found in the ventral, lateral and Pos and also in the MD tended to be more sparsely distributed.

After the tracer injection into the dorsal part of the $\mathrm{CPu}$ (Fig. 2), marked retrograde labeling was seen in the intralaminar nuclei, especially in the centrolateral (CL) nucleus. Labeled neurons in the parafascicular (PF) nucleus were located mainly in its lateral and dorsal parts. Labeled neurons in the PF tended to be located in its lateral and dorsal regions. A moderate to small number of labeled neurons were observed in the ethmoid (Eth), MD, VL and VM nuclei. After FG injection into the ventromedial $\mathrm{CPu}$ regions including the $\mathrm{Acb}$ (cases 97024 and 97039 in Figs. 1 and 2), retrograde labeling was marked in the midline nuclei, especially in the paraventricular (PV) and intermediodorsal nuclei (IMD), and less marked in the reunions ( $R e$ ) and rhomboid ( $\mathrm{Rh}$ ) nuclei. A moderate number of labeled cells were found in the rostral intralaminar thalamic nuclei, and particularly in the central medial (CM) nucleus. Labeled neurons in the PF were distributed in its medial portion. The ventral, posterior and lateral thalamic nuclear groups had no labeled neurons in these cases. The MD contained a moderate number of labeled neurons in the medial portion adjacent to the midline thalamic nuclei. FG injections in the ventral part of $\mathrm{CPu}$ resulted in neuronal labeling in the midline and intralaminar thalamic nuclei (Fig. 5B).

After FG injection into the medial part of the $\mathrm{CPu}$ (Fig. 3), moderate labeling was seen in the midline nuclei, mainly in the dorsal part of these nuclei. Labeled neurons were observed lateral to the stria medullaris in the CL, LD and LP. As medial injections were located more ventrally (cases 97027 or 98001), higher degrees of labeling were observed in the midline thalamic nuclei. After FG injections in the lateral CPu (Fig. 3), labeled neurons were distributed within the ventral portions of the rostral and caudal intralaminar nuclei (CM, CL, PC and PF). A moderate to high number of labeled neurons were found in the VM, VL and Po. 
After FG injections in the rostral pole of the $\mathrm{CPu}$ (Fig. 4), retrogradely labeled neurons were mainly found in the rostral regions of the midline and intralaminar thalamic nuclei. A moderate number of labeled neurons were seen in the MD, VM, VL and VA. Neurons in the PF were concentrated in its rostral pole. Caudal FG injections in the $\mathrm{CPu}$ (Fig. 4) resulted in a small to moderate number of labeled neurons in the caudal regions of the midline and intralaminar thalamic nuclei. A moderate to large number of labeled neurons were found within the VPM and Po.

When two retrograde tracers were injected in the dorsomedial, rostrocaudal and mediolateral regions of the $\mathrm{CPu}$, no double-labeled neurons were detected (Fig. 5). In these three cases, neurons labeled with FG or CTB were observed abundantly in the midline and intralaminar thalamic nuclei and less abundantly in the VM, VL and VA. Thus, in case 98085 (Fig. 5), the FG-labeled neurons appeared to be distributed in the ventromedial regions of the $\mathrm{CM}, \mathrm{PC}$ and $\mathrm{PF}$, whereas the CTB-labeled neurons were found in the dorsolateral regions of the same thalamic nuclei and also in the dorsal zone of the CL. Furthermore, when the two types of retrogradely labeled neurons were present in the $\mathrm{CL}$ and PC, clusters of neurons labeled with FG were intermingled with those labeled with CTB (Fig. 5). In case 98050 , two injections were made in the $\mathrm{CPu}$; FG was injected into the ventromedial region of the rostral part of the $\mathrm{CPu}$, including the dorsal part of the Acb, while CTB was injected more laterally and dorsally (Fig. 5). Many FG-labeled neurons were observed not only in the midline nuclei, but also in the region
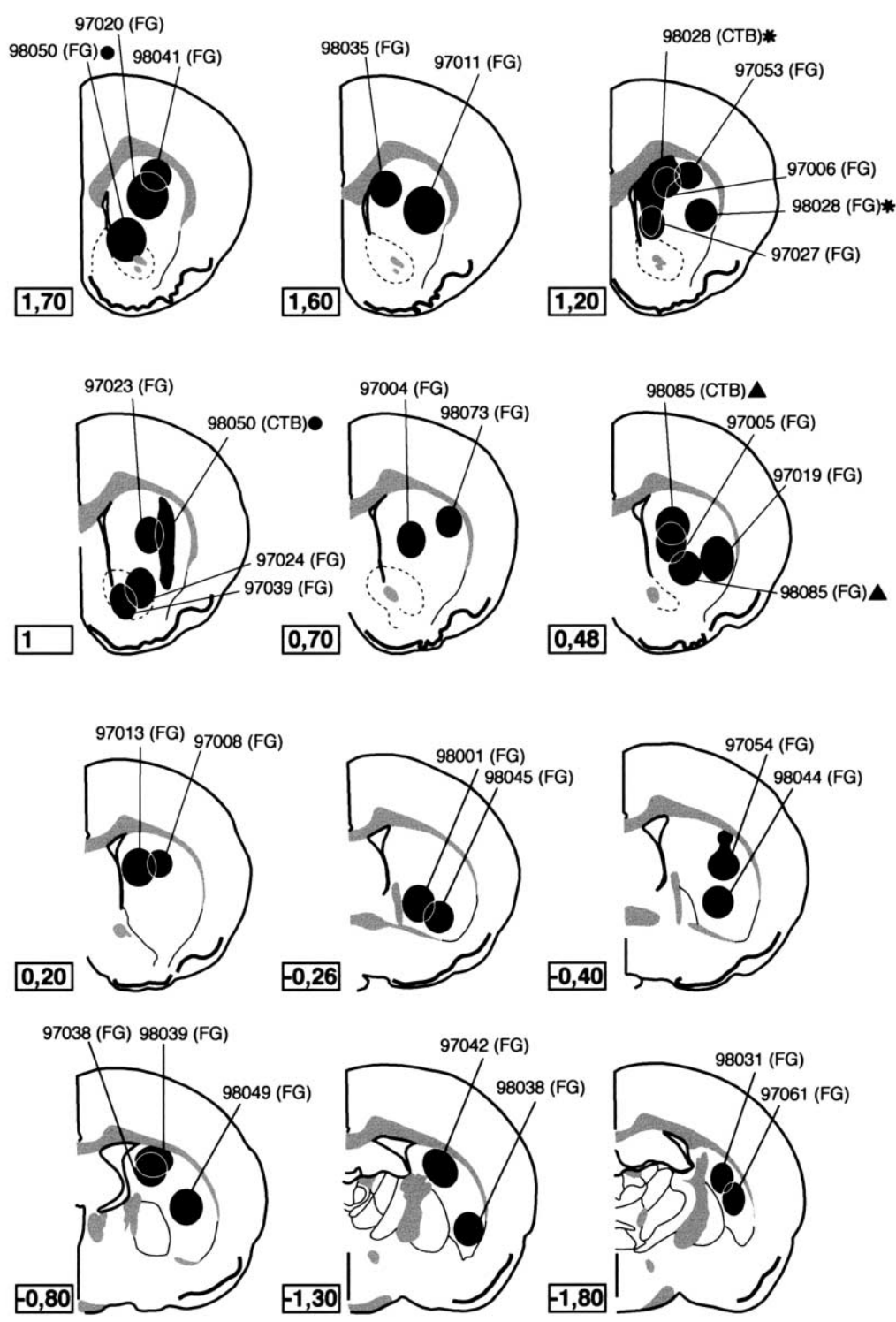

Fig. 1. Schematic drawings showing the location of the FG and CTB injection sites in coronal sections of the CPu as well as the distance from the bregma of each section. Asterisks, filled circles and triangles represent the cases in which FG was injected concomitantly with CTB. 
97053

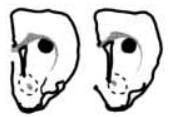

A

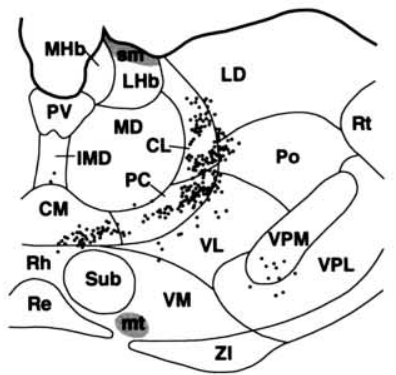

B

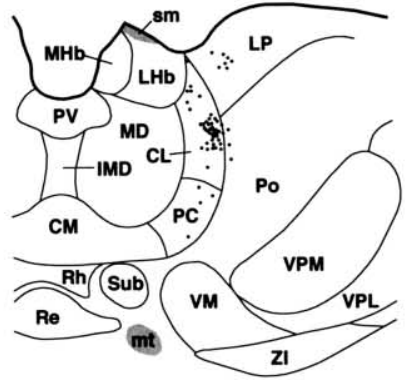

C

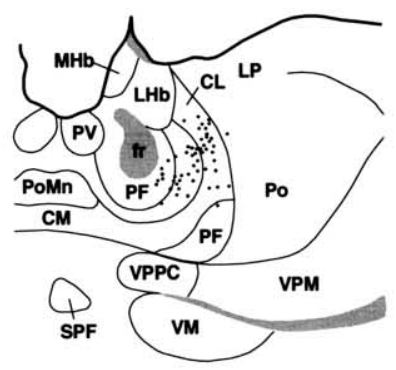

D

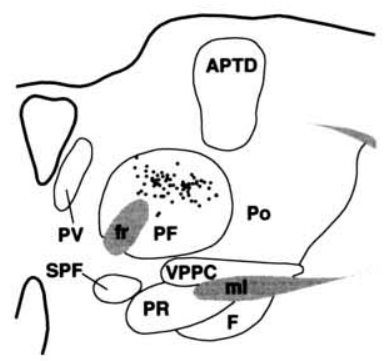

97024

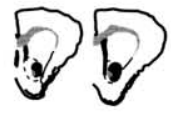

$A^{\prime}$

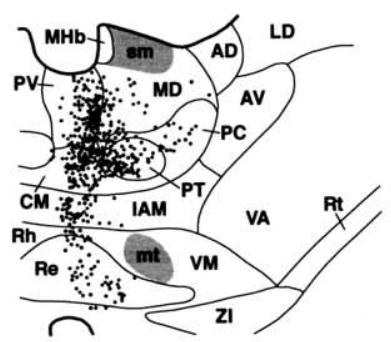

B'

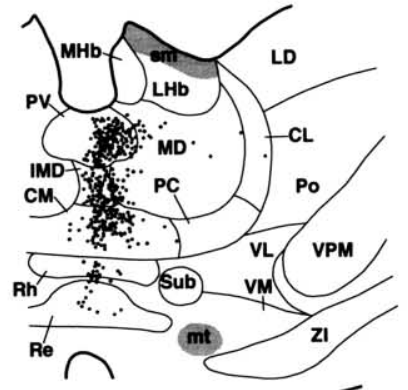

C'

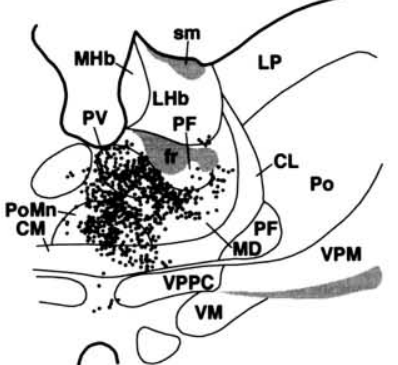

D'

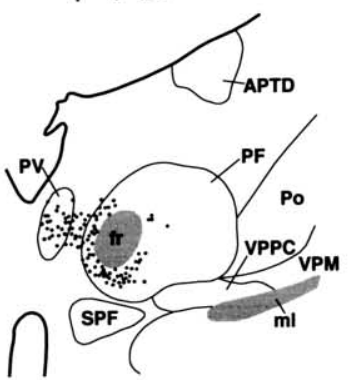

Fig. 2. Schematic drawings showing two examples of the organization of the thalamostriatal neurons in the rat projecting to different regions of the $\mathrm{CPu}$, after single injections of the retrograde tracer, FG. Left (A-D) four coronal sections through the thalamus, arranged rostrocaudally showing retrogradely labeled neurons from a dorsal injection in the $\mathrm{CPu}$, shown at the top of this column (case 97053$)$. Right $\left(\mathrm{A}^{\prime}-\mathrm{D}^{\prime}\right)$ four coronal sections through the thalamus, arranged rostrocaudally showing retrogradely labeled neurons from a ventral injection in the Acb, shown at the top of this column (case 97024 ). Note the clear lateromedial topography displayed by thalamic cells projecting to dorsal versus ventral regions of the $\mathrm{CPu}$. Each dot represents one labeled neuron. Stippled areas correspond to fiber tracts.

lateral to the stria medullaris, which includes the CL and LD and in the rostral pole of the PF. In contrast, CTB-labeled neurons were found in the ventrolateral regions of the rostral intralaminar nuclei and in the caudal region of the PF. The area of distribution of FG-labeled neurons overlapped with that of CTB-labeled neurons only in the CM. Finally, in case 98028 (Fig. 5), thalamostriatal neurons displayed a distribution characterized by several common features of the two aforementioned patterns of projections.

The main results of the present study are:

1. Retrogradely labeled cells were found in the midline and intralaminar thalamic nuclei in all cases included in this study. Labeled neurons were further seen in the ventral, lateral and Pos in the rats injected with the tracer into $\mathrm{CPu}$, but not in the rats injected with the tracer into the ventromedial $\mathrm{CPu}$ regions including the Acb. 
2. Topographical organization was observed in the projections from the midline-intralaminar thalamic nuclei to the $\mathrm{CPu}$. After the tracer injection into the dorsal part of the $\mathrm{CPu}$ or the ventral part of $\mathrm{CPu}$ (including the Acb), labeled neurons in the midlineintralaminar thalamic nuclei were distributed predominantly in the lateral part of the intralaminar nuclei or the midline nuclei, respectively. On the
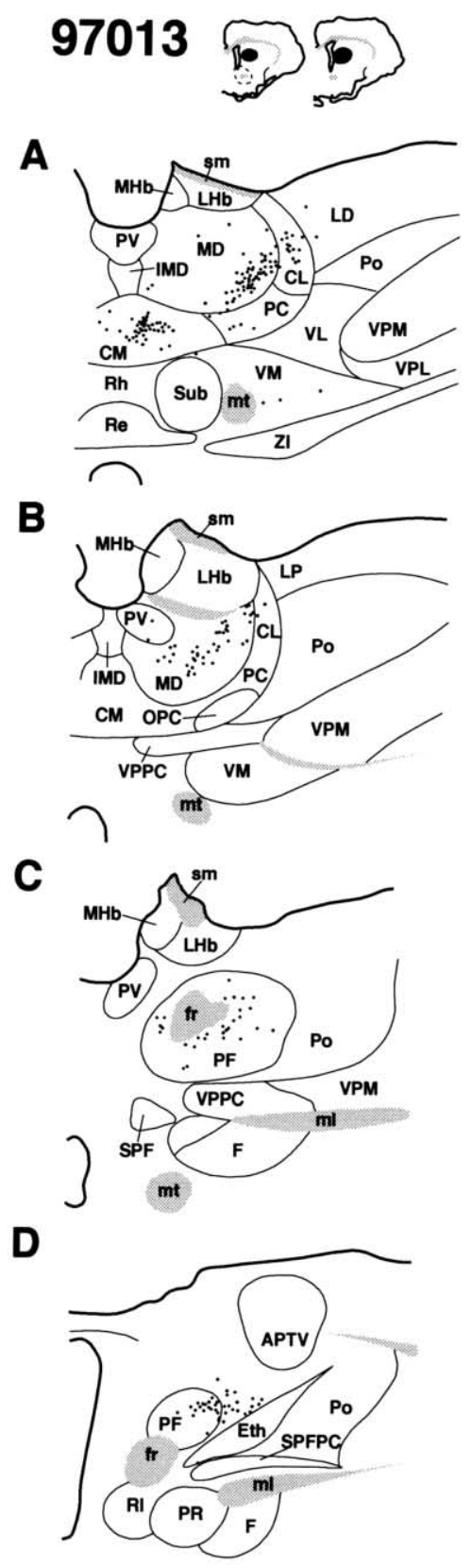

Fig. 3. Schematic drawings showing two examples of the organization of the thalamostriatal neurons in the rat projecting to different regions of the $\mathrm{CPu}$, after single injections of the retrograde tracer FG. Left (A-D) four coronal sections through the thalamus, arranged rostrocaudally showing retrogradely labeled neurons from a medial injection in the $\mathrm{CPu}$, shown at the top of this column (case 97013). Right $\left(\mathrm{A}^{\prime}-\mathrm{D}^{\prime}\right)$ four coronal sections through the thalamus, arranged rostrocaudally showing retrogradely labeled neurons from a lateral injection in the $\mathrm{CPu}$, shown at the top of this column (case 97019 ). Note the certain dorsoventral topography displayed by thalamic cells projecting to medial versus lateral regions of the $\mathrm{CPu}$. Each dot represents one labeled neuron. Stippled areas correspond to fiber tracts.

other hand, after the tracer injection into the medial or the lateral part of the $\mathrm{CPu}$, labeled neurons in the midline-intralaminar nuclei were distributed mainly in the dorsal or the ventral part of these nuclei, respectively.

3. Topographical organization was also observed in the thalamostriatal projections from the ventral and Pos. After the tracer injection into the rostral part
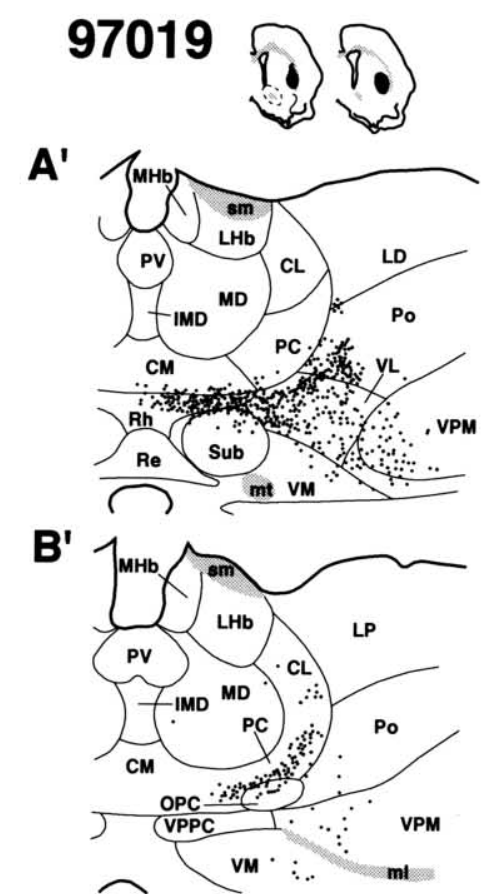

C'

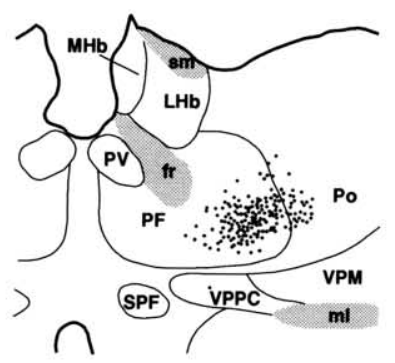

D'

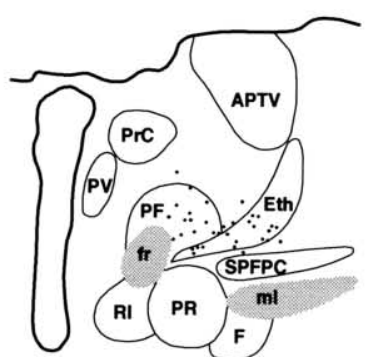


97020

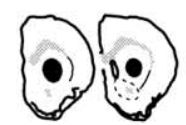

A

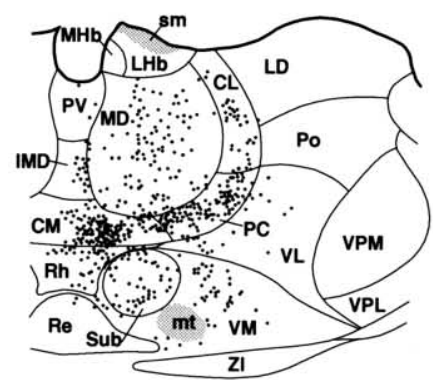

B

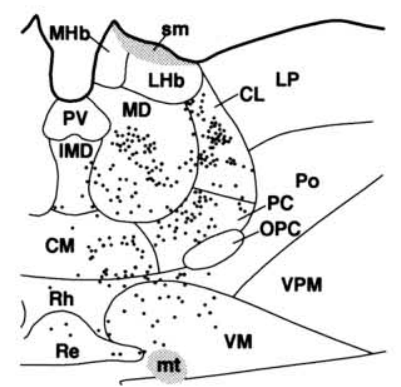

C

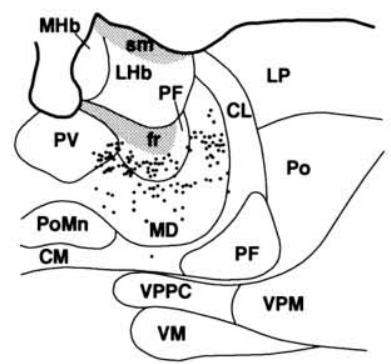

D

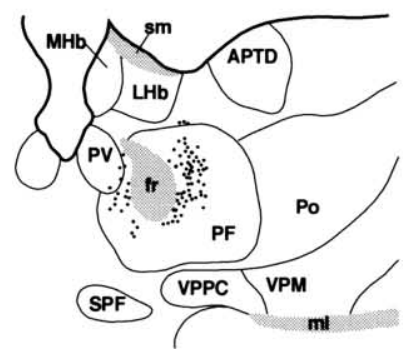

97061
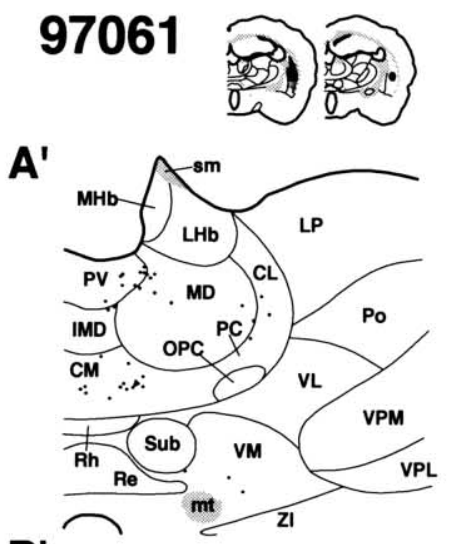

B'

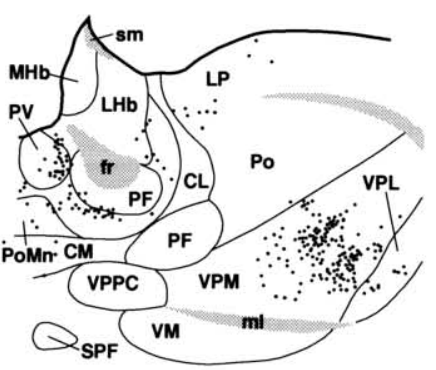

C'

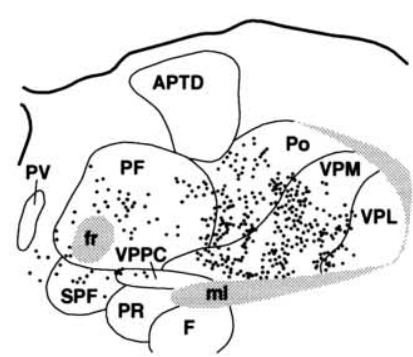

D'

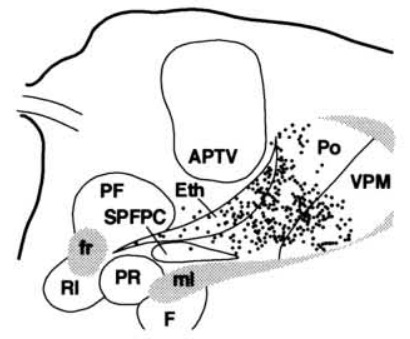

Fig. 4. Schematic drawings showing two examples of the organization of the thalamostriatal neurons in the rat projecting to different regions of the $\mathrm{CPu}$, after single injections of the retrograde tracer FG. Left (A-D) four coronal sections through the thalamus, arranged rostrocaudally showing retrogradely labeled neurons from a rostral injection in the $\mathrm{CPu}$, shown at the top of this column (case 97020$)$. Right $\left(\mathrm{A}^{\prime}-\mathrm{D}^{\prime}\right)$ four coronal sections through the thalamus, arranged rostrocaudally showing retrogradely labeled neurons from a caudal injection in the $\mathrm{CPu}$, shown at the top of this column (case 97061). Note the clear rostrocaudal topography displayed by thalamic cells projecting to rostral versus caudal regions of the $\mathrm{CPu}$. Each dot represents one labeled neuron. Stippled areas correspond to fiber tracts.

of the $\mathrm{CPu}$, labeled neurons were distributed mainly in the rostral part of the ventral nuclear group. On the other hand, after the tracer injection into the caudal part of the $\mathrm{CPu}$, labeled neurons were distributed mainly in the caudal part of the ventral nuclear group as well as in the posterior nuclear group.

\section{Discussion}

\subsection{Origin and organization of the thalamostriatal projections}

Thalamostriatal neurons were always found in the midline and intralaminar thalamic nuclei after injec- 
tions of retrograde tracers in different regions of the $\mathrm{CPu}$. These findings are in agreement with those of previous anatomical studies in the rat (Nauta et al.,
1974; Newman and Winans, 1980; Veening et al., 1980; Hazlett and Bagley, 1983; Phillipson and Griffiths, 1985; Fuller et al., 1987; Christie et al., 1987; Berendse
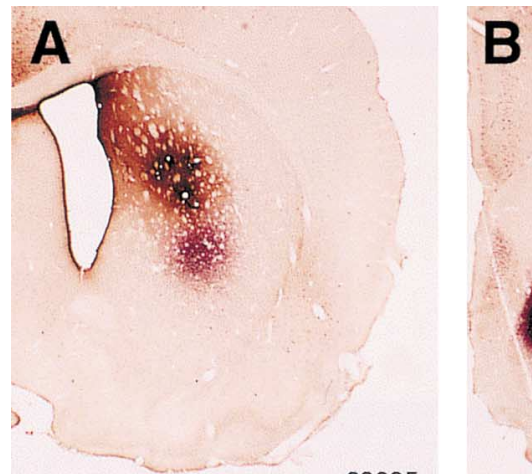

98085

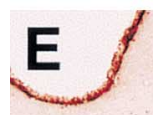

PV

MiD

CL
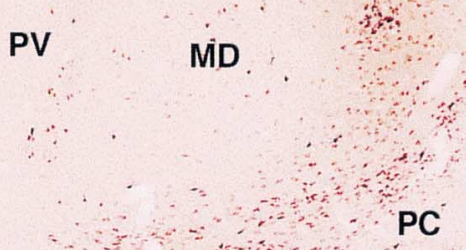

CM

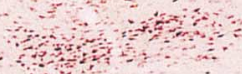

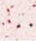

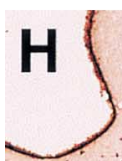

98085

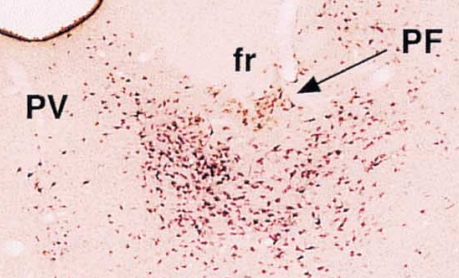

MD

98085

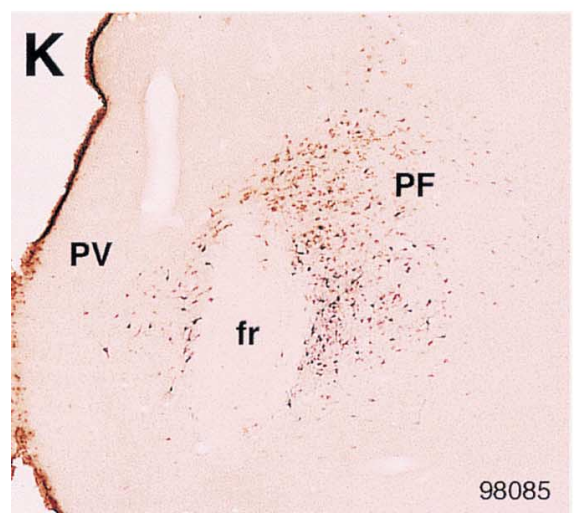

C

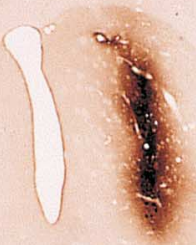

98050
D

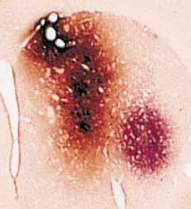

98050

98028
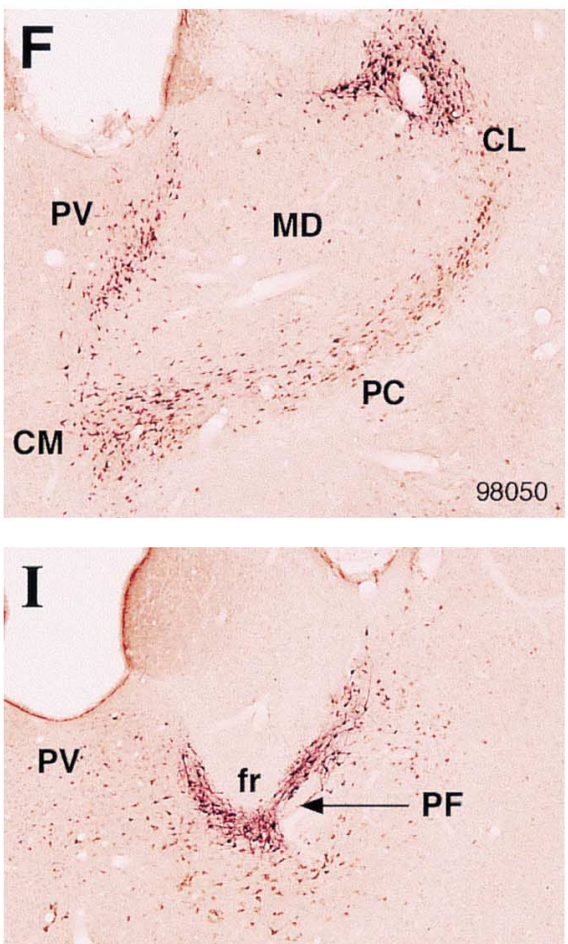

MD

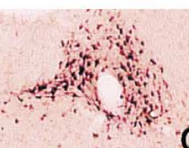

CL 98050

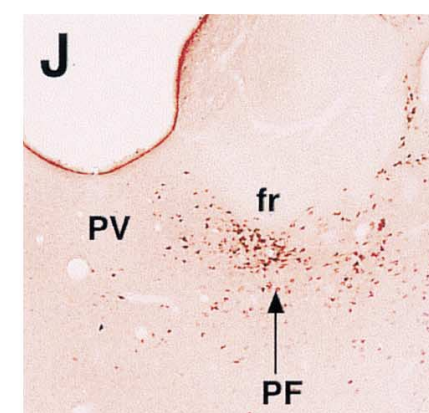

98050

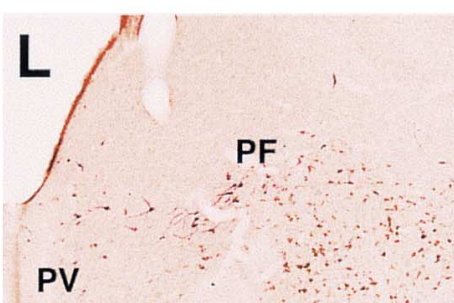

fr

98050

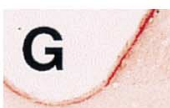

PV

MD

$\mathrm{CL}$

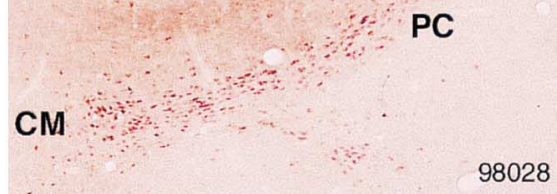

98028

98028

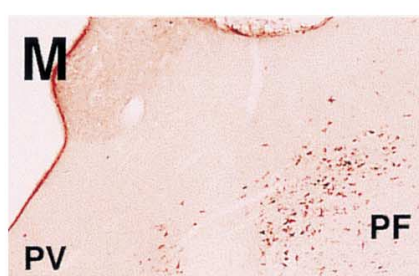

fr

98028

Fig. 5. 
and Groenewegen, 1990). Our work demonstrates labeled neurons in the Re. This projection has already been mentioned in some studies (Newman and Winans, 1980; Phillipson and Griffiths, 1985; Christie et al., 1987; Ohtake and Yamada, 1989) but has not been reported in other studies (Veening et al., 1980; Berendse and Groenewegen, 1990).

The VM, VL and VA contained a moderate number of retrogradely labeled neurons after FG injections in the ventrolateral areas of the rostral $\mathrm{CPu}$. The projection from the ventral thalamic nuclei has been described in the rat (Jones and Leavitt, 1974; Veening et al., 1980; Berendse and Groenewegen, 1990), cat (Royce, 1978; Beckstead, 1984a; Takada et al., 1985; Jarayaman, 1985; Heras et al., 1998a,b, 1999), dog (Tanaka et al., 1986) and primates (Parent et al., 1983; Smith and Parent, 1986; Nakano et al., 1990). Furthermore, the CL, LD and LP were found to project to the medial territories of the $\mathrm{CPu}$ in agreement with previous reports (Phillipson and Griffiths, 1985; Funaki et al., 1998). The projection from the Po, Eth and the posteromedian thalamic nucleus (PoMn) has already been described in the rat (Veening et al., 1980; Lin et al., 1984), cat (Beckstead, 1984b; Takada et al., 1985; Jarayaman, 1985) and primates (Lin et al., 1984).

There is some discrepancy in the literature in the projection from the MD to the striatum. The projection to the Acb from the MD has been described in the rat by several authors (Veening et al., 1980; Christie et al., 1987; Deschênes et al., 1995), although this projection has not been confirmed by others (van der Kooy, 1979). There are contradictory findings about this projection in the cat (Macchi et al., 1984; Sato et al., 1979; Beckstead, 1984a; Heras et al., 1998a) and primates (Nakano et al., 1990; Giménez-Amaya et al., 1995).

A considerable amount of labeled neurons were found in the VPM and VPL after FG injections in the postcommisural $\mathrm{CPu}$. These projections have not been generally accepted but experiments in our laboratory with anterograde tracers have confirmed their existence (Erro et al., 2001).

The topography is, in broad terms, in agreement with that described in other studies (van der Kooy, 1979; Berendse and Groenewegen, 1990). We can deduce from our retrograde tracer study that the terminal projection fields in the $\mathrm{CPu}$ of the different midline and intralaminar thalamic nuclei have the distribution described by Berendse and Groenewegen (1990), but with overlapping areas between two adjacent terminal fields. Furthermore, our results demonstrate that the rostral part of a midline or intralaminar nuclear groups projects more densely to a rostral part in the striatum and vice versa (Veening et al., 1980; Berendse and Groenewegen, 1990).

\subsection{Functional implications}

Midline and intralaminar thalamic nuclei were initially considered to be the source of a diffuse and unspecific projection system to the cortex and striatum (see references in Jones, 1985; Macchi and Bentivoglio, 1986; Steriade et al., 1997). These nuclei may act in parallel over the cerebral cortex and the striatum by means of collaterals of thalamic neurons (Cesaro et al., 1985; Deschênes et al., 1996a,b; Otake and Nakamura, 1998). On the other hand, the restricted and striatal fields of termination of thalamocortical fibers arising from the midline and intralaminar thalamic nuclei have also been reported (Itoh and Mizuno, 1977; Berendse and Groenewegen, 1990, 1991). In the present study, we observed the topographical arrangement of thalamostriatal neurons in the midline and intralaminar thalamic nuclei. These thalamostriatal projections from the midline and intralaminar thalamic nuclei may possible participate in the feed back circuits between the input and the output systems of the basal ganglia (Heras de las et al., 1998b; Erro et al., 1999; Lanciego et al., 1999; Mengual et al., 1999; Giménez-Amaya et al., 2000).

Little is known about the position of the thalamostriatal projections on the general scheme of the organization of the basal ganglia (Mengual et al., 1999; Giménez-Amaya et al., 2000). Physiological and ultrastructural studies reveal the specific role of these projections (Dubé et al., 1988; Lapper and Bolam, 1992; Consolo et al., 1996). Thus, Matsumoto et al. (2001) have suggested very recently that neurons in the CM and PF supply striatal neurons with information about behaviorally significant sensory events that can activate conditional responses of striatal neurons in combination with dopamine-mediated nigrostriatal inputs having motivational value. Furthermore, the variety of the

Fig. 5. Photomicrographs showing three examples of the distribution of retrogradely labeled neurons in the rat thalamus after the injections of FG and CTB in two different sites of the $\mathrm{CPu}$ in the same animal. (A), Coronal section through the precommissural $\mathrm{CPu}$ illustrating the $\mathrm{CTB}$ injection site stained brown with DAB ventrally, the FG injection site stained purple with V-VIP for case 98085 . (B), Coronal section through the rostral $\mathrm{CPu}$ showing the FG injection site located in its ventral portion and in Acb of case 98050 . (C) A caudal level of the same case showing the CTB injection site in the $\mathrm{CPu}$. (D) Coronal section through the precommissural $\mathrm{CPu}$ showing the CTB (dorsomedial) and FG (ventrolateral) injection sites of case 98028 . (E-G) Coronal sections through the rostral thalamus showing the different spatial organization of CTB and FG retrogradely labeled neurons in some midline (PV) and rostral intralaminar (CL, CM and PC) thalamic nuclei. (H-J) Coronal sections through the caudal thalamus showing the different distribution of the thalamostriatal neurons in the PV thalamic nucleus, in the caudal portion of the MD thalamic nucleus and in the rostral pole of the PF thalamic nucleus. $(\mathrm{K}-\mathrm{M})$ Coronal sections through the caudal thalamus showing the topography of thalamostriatal neurons in the caudal portion of PF thalamic nucleus. Scale bar, (A-D) $3000 \mu \mathrm{m}$; (E-M) $750 \mu \mathrm{m}$. 
termination patterns of the thalamostriatal projections is widened when the compartmental striatal chemoarchitecture is taken into account (Herkenham and Pert, 1981). Anterograde studies in the cat (Ragsdale and Graybiel, 1991) as well as in primates (Kalil, 1978; Sadikot et al., 1992) have shown that the striatal input from the thalamus resulted in a clear mosaicism in their termination in the matrix compartment. Recently, a new concept for the organization of the basal ganglia has emerged based on results of various experimental and clinical studies. The basal ganglia are no longer considered as a unidirectional vertical system, and it has been proposed that they are a highly organized network where internal or horizontal circuits play an important role in the modulation of their final outcome (Obeso et al., 2000). The thalamostriatal projections, particularly those arising from caudal intralaminar nuclei, may represent an important horizontal loop in the internal circuits of the basal ganglia.

\section{Acknowledgements}

The authors would like to thank Angeles Erdozain, Pedro García and Ainhoa Moreno for their technical assistance. This study was supported by CICYT PM980035, Departamento de Salud del Gobierno de Navarra, Fundación Marcelino Botín and Universidad de Navarra, and FIS 01/0237.

\section{References}

Beckstead, R.M., 1984a. The thalamostriatal projection in the cat. J. Comp. Neurol. 223, 313-346.

Beckstead, R.M., 1984b. A projection to the striatum from the medial subdivision of the posterior group of the thalamus in the cat. Brain Res. 300, 351-356.

Berendse, H.W., Groenewegen, H.J., 1990. Organization of thalamostriatal projection in the rat, with special emphasis on the ventral striatum. J. Comp. Neurol. 299, 187-228.

Berendse, H.W., Groenewegen, H.J., 1991. Restricted cortical termination fields of the midline and intralaminar thalamic nuclei in the rat. Neuroscience 42, 73-102.

Cesaro, P., Nguyen-Legros, J., Pollin, B., Laplane, S., 1985. Single intralaminar thalamic neurons project to cerebral cortex, striatum and nucleus reticularis thalami. A retrograde anatomical study in the rat. Brain Res. 325, 29-37.

Christie, M.J., Summers, R.J., Stephenson, J.A., Cook, C.J., Beart, P.M., 1987. Excitatory amino acid projections to the nucleus accumbens septi in the rat: a retrograde transport study utilizing $\mathrm{D}\left[{ }^{3} \mathrm{H}\right]$ aspartate and $\left[{ }^{3} \mathrm{H}\right] \mathrm{GABA}$. Neuroscience 22, 425-439.

Consolo, S., Baronio, P., Guidi, G., Di Chiara, G., 1996. Role of the parafascicular thalamic nucleus and $N$-methyl-D-aspartate transmission in the $\mathrm{D}_{1}$-dependent control of in vivo acetylcholine release in rat striatum. Neuroscience $71,157-165$.

Deschênes, M., Bourassa, J., Parent, A., 1995. Two different types of thalamic fibers innervate the rat striatum. Brain Res. 701, 288292.
Deschênes, M., Bourassa, J., Doan, V.D., Parent, A., 1996a. A single cell study of the axonal projections arising from the posterior intralaminar thalamic nuclei in the rat. Eur. J. Neurosci. 8, 329-343.

Deschênes, M., Bourassa, J., Parent, A., 1996b. Striatal and cortical projections of single neurons from the central lateral thalamic nucleus in the rat. Neuroscience 72, 679-687.

Dubé, L., Smith, A.D., Bolam, J.P., 1988. Identification of synaptic terminals of thalamic or cortical origin in contact with distinct medium-size spiny neurons in the rat neostriatum. J. Comp. Neurol. 267, 455-471.

Erro, E., Lanciego, J.L., Giménez-Amaya, J.M., 1999. Relationship between thalamostriatal neurons and pedunculopontine projections to the thalamus: a neuroanatomical tract-tracing study in the rat. Exp. Brain Res. 217, 162-170.

Erro, E., Lanciego, J.L., Arribas, J., Giménez-Amaya, J.M., 2001. Striatal inputs from the ventrobasal complex of the rat thalamus. Histochem. Cell Biol. 115, 447-454.

Fuller, T.A., Russchen, F.T., Price, J.L., 1987. Sources of presumptive glutamatergic/aspartergic afferents to the rat ventral striatopallidal region. J. Comp. Neurol. 258, 317-338.

Funaki, S., Meguro, R., Abe, H., Norita, M., 1998. The organization of the thalamostriatal projection from the lateral posterior thalamic nuclear complex (LP) in the pigmented rat. Neurobiology 6, $272-294$.

Geneser-Jensen, F.A., Blackstad, T.W., 1971. Distribution of acetyl cholinesterase in the hippocampal region of the guinea pig I. Entorhinal area, parasubiculum, and presubiculum. Z. Zellforsch. Mikrosk. Anat. 114, 460-481.

Giménez-Amaya, J.M., Scarnati, E., 1999. The thalamus as a place for interaction between the input and the output systems of the basal ganglia: a commentary. J. Chem. Neuroanat. 16, 149-152.

Giménez-Amaya, J.M., McFarland, N.R., de las Heras, S., Haber, S.N., 1995. Organization of thalamic projections to the ventral striatum in the primate. J. Comp. Neurol. 354, 127-149.

Giménez-Amaya, J.M., de las Heras, S., Erro, E., Mengual, E., Lanciego, J.L., 2000. Considerations on the thalamostriatal system with some functional implications. Histol. Histopathol. 15, $1285-1292$.

Graybiel, A.M., Ragsdale, C.W. Jr, 1978. Histochemically distinct compartments in the striatum of human, monkey, and cat demonstrated by acetylcholinesterase staining. Proc. Natl. Acad. Sci. USA $75,5723-5726$.

Groenewegen, H.J., 1988. Organization of the afferent connections of the mediodorsal thalamic nucleus in the rat, related to the mediodorsal-prefrontal topography. Neuroscience 24, 379-431.

Hazlett, J.C., Bagley, S.D., 1983. Origin and topography of thalamocaudate projections in the opossum. Neurosci. Lett. 36, 19-24.

de las Heras, S., Mengual, E., Velayos, J.L., Giménez-Amaya, J.M., 1997. New data on the anatomical organization of the thalamostriatal projections. Adv. Neurol. 74, 69-81.

de las Heras, S., Mengual, E., Velayos, J.L., Giménez-Amaya, J.M., 1998a. Re-examination of topographic distribution of thalamic neurons projecting to the caudate nucleus. A retrograde labeling study in the cat. Neurosci. Res. 31, 283-292.

de las Heras, S., Mengual, E., Giménez-Amaya, J.M., 1998b. Overlapping territories between the thalamostriatal and nigrothalamic projections in cats. Neuroreport 9, 1913-1916.

de las Heras, S., Mengual, E., Giménez-Amaya, J.M., 1999. Double retrograde tracer study of the thalamostriatal projections to the cat caudate nucleus. Synapse 32, 80-92.

Herkenham, M.Y., Pert, C.B., 1981. Mosaic distribution of opiate receptors, parafascicular projections and acetylcholinesterase in rat striatum. Nature 291, 415-418.

Itoh, K., Mizuno, N., 1977. Topographical arrangement of thalamocortical neurons in the centrolateral nucleus (CL) of the cat, with special reference to a spino-thalamo-motor cortical path through the CL. Exp. Brain Res. 30, 471-480. 
Jones, E.G., 1985. The Thalamus. Plenum Press, New York.

Jones, E.G., Leavitt, R.Y., 1974. Retrograde axonal transport and the demonstration of non-specific projections to the cerebral cortex and the striatum from thalamic intralaminar nuclei in the rat, cat and monkey. J. Comp. Neurol. 154, 349-378.

Kalil, K., 1978. Patch-like termination of thalamic fibers in the putamen of the rhesus monkey: an autoradiographic study. Brain Res. 140, 333-339.

Lanciego, J.L., Erro, E., Arribas, J., Giménez-Amaya, J.M., 1999. Output and input circuits of the primate basal ganglia: the relationship between the pallidothalamic projection and identified thalamostriatal neurons. Soc. Neurosci. Abstr. 25, 1924.

Lapper, S.R., Bolam, J.P., 1992. Input from the frontal cortex and the parafascicular nucleus to cholinergic interneurons in the dorsal striatum of the rat. Neuroscience 51, 533-545.

Lin, C.-S., May, P.J., Hall, W.C., 1984. Nonintralaminar thalamostriatal projections in the gray squirrel (Sciureus carolinensis) and tree shrew (Tupaia glis). J. Comp. Neurol. 230, 33-46.

Macchi, G., Bentivoglio, M., 1986. The intralaminar nuclei and the cerebral cortex. In: Jones, E.G., Peters, A. (Eds.), Sensory-Motor Areas and Aspects of Cortical Connectivity, Cerebral Cortex, vol. 5, Plenum Press, New York, pp. 355-401.

Macchi, G., Bentivoglio, M., Molinari, M., Minciacchi, D., 1984. The thalamo-caudate versus thalamo-cortical projections as studied in the cat with retrograde double labeling. Exp. Brain Res. 54, 225-239.

Mengual, E., de las Heras, S., Erro, E., Lanciego, J.L., GiménezAmaya, J.M., 1999. Thalamic interaction between the input and the output systems of the basal ganglia. J. Chem. Neuroanat. 16, $187-200$.

Matsumoto, N., Minamimoto, T., Graybiel, A.M., Kimura, M., 2001. Neurons in the thalamic CM-PF complex supply striatal neurons with information about behaviorally significant sensory events. J. Neurophysiol. 85, 960-976.

Nakano, K., Hasegawa, Y., Tokushige, A., Nakagawa, S., Kayahara, T., Mizuno, N., 1990. Topographical projections from the thalamus, subthalamic nucleus and pedunculopontine tegmental nucleus to the striatum in the Japanese monkey, Macaca fuscata. Brain Res. 4537, 54-68.

Nauta, H.J.W., Pritz, M.B., Lasek, R.J., 1974. Afferents to the rat caudoputamen studied with horseradish peroxidase. An evaluation of a retrograde neuroanatomical research method. Brain Res. 67, 219-238.

Newman, R., Winans, S.S., 1980. An experimental study of the ventral striatum of the golden hamster. I. Neuronal connections of the nucleus accumbens. J. Comp. Neurol. 191, 167-192.

Obeso, J.A., Rodríguez-Oroz, M.C., Rodríguez, M., Lanciego, J.L., Artieda, J., Gonzalo, N., Olanow, C.W., 2000. Pathophysiology of the basal ganglia in Parkinson's disease. Trends Neurosci. 23, $8-19$.

Ohtake, T., Yamada, H., 1989. Efferent connections of the nucleus reuniens and the rhomboid nucleus in the rat: an anterograde PHA-L tracing study. Neurosci. Res. 6, 556-568.

Otake, K., Nakamura, Y., 1998. Single midline thalamic neurons projecting to both the ventral striatum and the prefrontal cortex in the rat. Neuroscience 86, 635-649.

Parent, A., Mackey, A., De Bellefeuille, L., 1983. The subcortical afferents to caudate nucleus and putamen in primate: a fluorescence retrograde double labeling study. Neuroscience 10, 11371150.

Paxinos, G., Watson, C., 1998. The rat brain in stereotaxic coordinates. Academic Press, Sydney, Australia.

Phillipson, O.T., Griffiths, A.C., 1985. The topographic order of inputs to nucleus accumbens in the rat. Neuroscience 16, 275296.

Ragsdale, C.W. Jr, Graybiel, A.M., 1991. Compartmental organization of the thalamostriatal connection in the cat. J. Comp. Neurol. 311, 134-167.

Rosene, D.L., Roy, N.J., Davis, B.J., 1986. A cryoprotection method that facilitates cutting frozen sections of whole monkey brains for histological and histochemical processing without freezing artifact. J. Histochem. Cytochem. 34, 1301-1316.

Royce, G.J., 1978. Cells of origin of subcortical afferents to the caudate nucleus: a horseradish peroxidase study in the cat. Brain Res. 153, 465-475.

Sadikot, A.F., Paren, A., Smith, Y., Bolam, J.P., 1992. Efferent connections of the centromedian and parafascicular thalamic nuclei in the squirrel monkey: a light and electron microscopic study of the thalamostriatal projection in relation to striatal heterogeneity. J. Comp. Neurol. 320, 228-242.

Sato, M., Itoh, K., Mizuno, N., 1979. Distribution of thalamo-caudate neurons in the cat as demonstrated by horseradish peroxidase. Exp. Brain Res. 34, 143-153.

Smith, Y., Parent, A., 1986. Differential connections of caudate nucleus and putamen in the squirrel monkey (Saimiri sciureus). Neuroscience 18, 347-371.

Steriade, M., Jones, E.G., McCormick, D.A., 1997. Thalamus. In: Organisation and Function, vol. I. Elsevier, Amsterdam.

Takada, M., Itoh, K., Yasui, Y., Sugimoto, T., Mizuno, N., 1985. Topographical projections from the posterior thalamic regions to the striatum in the cat, with reference to possible tecto-thalamostriatal connections. Exp. Brain Res. 60, 385-396.

Tanaka, D. Jr, Isaacson, L.G., Trosko, B.K., 1986. Thalamostriatal projections from the ventral anterior nucleus in the dog. J. Comp. Neurol. 247, 56-68.

van der Kooy, D., 1979. The organization of the thalamic, nigral and raphe cells projecting to the medial versus lateral caudate-putamen in rat. A fluorescent retrograde double labeling study. Brain Res. 169, 381-387.

Veening, J.G., Cornelissen, F.M., Lieven, P.A.J.M., 1980. The topical organization of the afferents to the caudatoputamen of the rat. A horseradish peroxidase study. Neuroscience 5, 1253-1268. 